\title{
Performance Analysis of Spectrum Sensing in Cognitive Radio under Low SNR and Noise
} Floor

\author{
P. Venkatapathi, Habibulla Khan, S. SrinivasaRao
}

\begin{abstract}
Cognitive radio (CR) is a new technology that is proposed to improve spectrum efficiency by allowing unlicensed secondary users to access the licensed frequency bands without interfering with the licensed primary users. As there are several methods available for spectrum sensing, the energy detection (ED) is more popular due to its simple implementation. However, ED is more vulnerable to the noise uncertainty so for that reason, we present a robust detector using signal to noise ratio (SNR) with dynamic threshold energy detection technique is combined with the kernel principal component analysis (KPCA) in Cognitive Radio Networks (CRN). The primary purpose of kernel function is to ensure that its dependency relies on inner-product of data without the feature space data requirement. In this paper, with the aid of kernel function the spectrum sensing with the leading eigenvector approach is modified to a feature space of higher dimensionality. By introducing of efficient detection system with dynamic threshold facility helps the better detection levels even low SNR values with quite a lot of noise uncertainty levels. The simulation results of the proposed system reveal that KPCA outperforms with that of traditional PCA in terms of false alarm rate, detector performance when tested under various uncertainties for orthogonal frequency division multiplexing signal.
\end{abstract}

Index Terms: Cognitive Radio; Energy Detection; kernel Principal Component Analysis; Spectrum sensing; Principal Component Analysis

\section{INTRODUCTION}

With the advances of information and communication technologies and the development of the world economy, there has been an explosive demand for wireless communication services. Specifically, wireless internet access through smart-phones, tablets, and laptops has become the primary means of personal communication. The wireless communication applications go beyond the personal communication services to perform sensing, monitoring system, patient monitoring, and vehicle network and so on.

Revised Manuscript Received on December 30, 2019.

* Correspondence Author

P. Venkatapathi*, Research Scholar, Department of ECE, KLEF, Vijayawada, Andhra Pradesh, India.

Dr. Habibulla Khan, Professor, Department of ECE, KLEF, Andhra Pradesh, India.

Dr. S. Srinivasa Rao, Professor \& HOD, Department of ECE, MRCET, Hyderabad, Telangana, India.

(C) The Authors. Published by Blue Eyes Intelligence Engineering and Sciences Publication (BEIESP). This is an open access article under the CC BY-NC-ND license (http://creativecommons.org/licenses/by-nc-nd/4.0/)
This proliferation of broad contact through the association of the network has become an integral part of various types of wireless communication devices, which already enable users to communicate with different services from remote operating areas of the available (and useful) radio spectrum. The need for wireless services in the communications industry has led engineers and professionals to focus a great deal on the rapid development of reliable wireless communications technologies. According to the views of [2], the spectrum distribution utilization variation is more useful to give the chance to users based on this regulatory committee. Referring to the Fig.1, most of the portion of bands are underutilized in certain cases and over-utilized in other cases. So, to fix this problem of scarcity in the allocation of frequency bands to the secondary users (SUs) which are not preoccupied with the primary users (PUs) without any interference. To alleviate the above issue of detection of channel occupancy, several authors worked on this theory as well implemented experimental testbeds, some of them are: In [3], the authors proposed a low complexity Energy detection (ED) scheme to detect the PU occupancy with low cost. Although the ED is a simpler mechanism fails to address the detection at lower signal-to-noise-ratios (SNR). In [4], the authors proposed a new framework for detecting the spectrum availability data and adopted intelligent-decision making method for sensing spectrum.

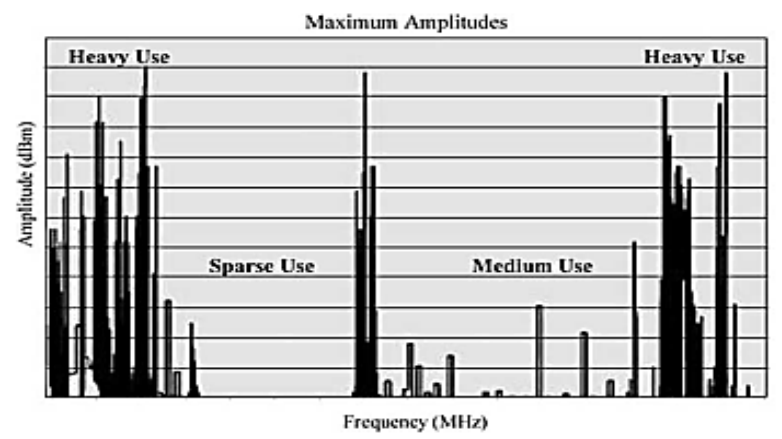

Fig.1. Signal strength distribution over the wireless spectrum [3]

Later in [5], the authors contributed to modify the multi-band spectrum sensing scheme with the aid of search heuristics to some extent. In [6], the authors investigated the problem of maximization that can solve the spectrum sensing duration over the complete period of the sensing cycle.

Published By:

Blue Eyes Intelligence Engineering

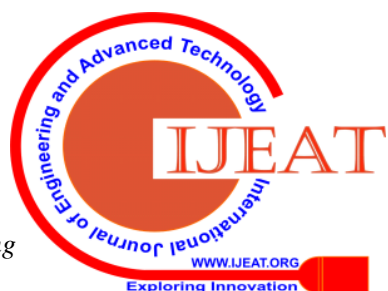


Also the work recommended the scheme for unknown constant signals for CR network, the cooperative sensing scheme, compared the various hard and soft energy-efficient schemes. Another important work on the cyclostationary feature detection approach was investigated by the authors in [7]. In this paper, PCA, KPCA is of particular relevance to efficient spectrum sensing and is examined along with the combination of the ED mechanism.

The main contributions and organization of this paper are summarized as follows: In section 2 we describe background details of different cognitive radio networks. The section 3 system model. The section 4 discusses about proposed system. The section 5 deliberates results and discussions. Finally, in section 6 we concluded the paper.

\section{BACKGROUND WORKS}

Cognitive radio technology allow the primary and secondary users to share the same spectrum by either Overlay technique, i.e., when white space is sensed, SU starts its transmission in that white space, or Underlay technique, i.e., SU share the spectrum by transmitting at the same time as the PU but at low power so as not to interfere with the PU. Cognitive radios have the following general model known as cognitive cycle shown in Fig.2 wherein it has the ability to sense spectrum with smart technology.

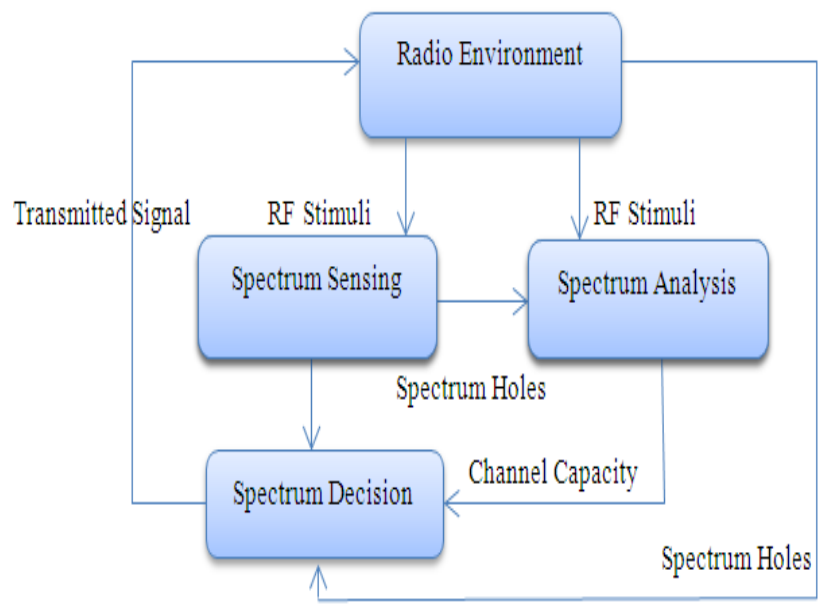

Fig.2. Cognitive Cycle

The primary understanding of the cognitive radio cycle is necessary because of its abrupt channel variations under noise uncertainties [11-13], as illustrated in figure.2 clearly, so one can address the decision of suitable algorithm for decision mechanism in the CR network.

\section{SYSTEM MODEL}

Let us consider the detection problem in this study can be characterized as a binary postulate as

$$
\begin{gathered}
H_{0}: Y[n]=W[n] \\
H_{1}: Y[n]=W[n]+h S[n] \\
\tau>\lambda \text { primary user present, } \\
\tau<\lambda \text { primary user absent }
\end{gathered}
$$

where $S$ treated as primary user signal, $Y$ be the secondary user signal, $W$ treated to AWGN and $h$ is the channel gain. The decision threshold representation $(\lambda)$ is useful largely because it allows the test statistic $(\tau)$ as

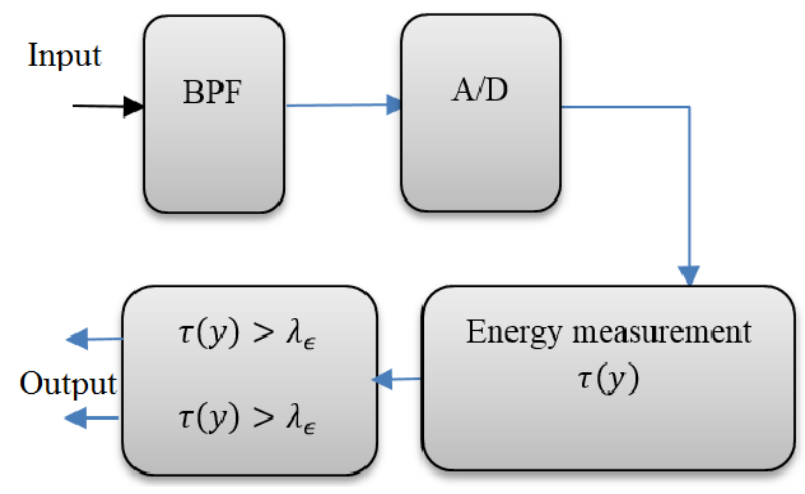

Fig. 3. Energy detector block diagram [9]

Referring to the Fig. 3 the simple Energy detector mechanism with two hypothesis can be put in mathematical form as [10].

$\tau(n)=\sum_{n=1}^{N-1}(Y(n))^{2}$

Where $Y(n)$ treated to be received signal, $N$ is the sample size, $\tau(n)$ considered as test statistic of energy.

According to the key concept of central limit theorem, the test data can be put in the form as

$H_{0}=T \sim \operatorname{Normal}\left(N \sigma_{w}^{2}, 2 N \sigma_{w}^{4}\right)$
$H_{1}=T \sim \operatorname{Normal}\left(N\left(\sigma_{w}^{2}+\sigma_{x}^{2}\right), 2 N\left(\sigma_{w}^{2}+\sigma_{x}^{2}\right)^{2}\right)$

$\mathrm{T}>\gamma$ signal is present

$\mathrm{T}<\gamma$ signal is absent

Then $P_{d}$ and $P_{f}$ can be calculated as:

$p_{d}=p\left(T>\gamma \mid H_{1}\right)=Q\left(\frac{\mathrm{Y}-N\left(\sigma_{w}^{2}+\sigma_{X}^{2}\right)}{\sqrt{2 N\left(\sigma_{w}^{2}+\sigma_{X}^{2}\right)^{2}}}\right)$
$p_{f a}=p\left(T>\gamma \mid H_{0}\right)=Q\left(\frac{\gamma-N \sigma_{w}^{2}}{2 N \sigma_{w}^{4}}\right)$

$Q(x)=\frac{1}{2 \pi \int_{X}^{\infty} \exp \left(\frac{-y^{2}}{2}\right) d y}$

We get the detection probability $P_{d}$ by suitably substituting the threshold value in the eq. (7). Another parameter minimum observation window $N_{\min }$ can be obtained by

$N_{\text {min }}=2\left[\left(Q^{-1}\left(P_{f a}\right)-Q^{-1}\left(P_{d}\right) S N R^{-1}-Q^{-1}\left(P_{d}\right)\right]^{2}\right.$

Where $S N R=\frac{\sigma_{X}}{\sigma_{w}}$

It was clearly shown from eq. (9), if the SNR value is minimum, then the signal will not be detected. Although the fixed threshold schemes are widely used in spectrum sensing for CR networks, they are inflexible with inaccurate decision making and their fluctuating environment.

Published By:

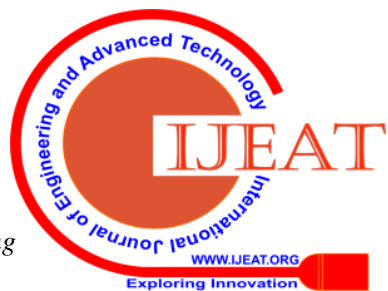


So, it results in higher miss detection and false alarm rates shows that its inefficiency to use for sensing spectrum. So, therefore, there is a requirement of focusing on mitigation sensing failure problem [8] by introducing a combined spectrum sensing method concentrated on the estimated SNR with an adaptive threshold in the combination of kernel PCA.

Probability of false alarm ( $P_{f a}$ ): When the signal is from the primary user, it is defined as the process of probability declaration of existence of PU signal during the period of spectrum vacancy(free) by the SUstated as,

$P_{f a}=P_{r}\left(H_{1} \backslash H_{0}\right)$

The lower the $P f a$, the additional the spectrum access the SUs will acquire.

Probability of misdetection $\left(\boldsymbol{P}_{\boldsymbol{m a}}\right)$ : It is defined as the process of probability declaration of non-existence of PU signal during the period of spectrum occupancy by the SU. It is defined as [15],

$P_{m d}=P_{r}\left(H_{0} \backslash H_{1}\right)$

Probability of detection $\left(\boldsymbol{P}_{\boldsymbol{d}}\right)$ : Another widely metrics is the probability of detection (PD) [14],

$P_{d}=1-P_{f a}$

$P_{d}=P_{r}\left(H_{1} \backslash H_{1}\right)$

Where $H_{0}$ and $H_{1}$ supposed to be the nonexistence and the existence of the PU signal. If there is $P d$ value to be high that indicates, the better the PU security is. For finding out the efficiency of the particular spectrum sensing methods, the three evaluation parameters are considered as

$P_{d}+P_{f a}+P_{m d}=1$

There have been confused region between the primary signal and noise variance. To detect the probability of false and miss detection parameters of the detector with the respect to various noise levels for spectrum sensing process as depicted in Fig.4.

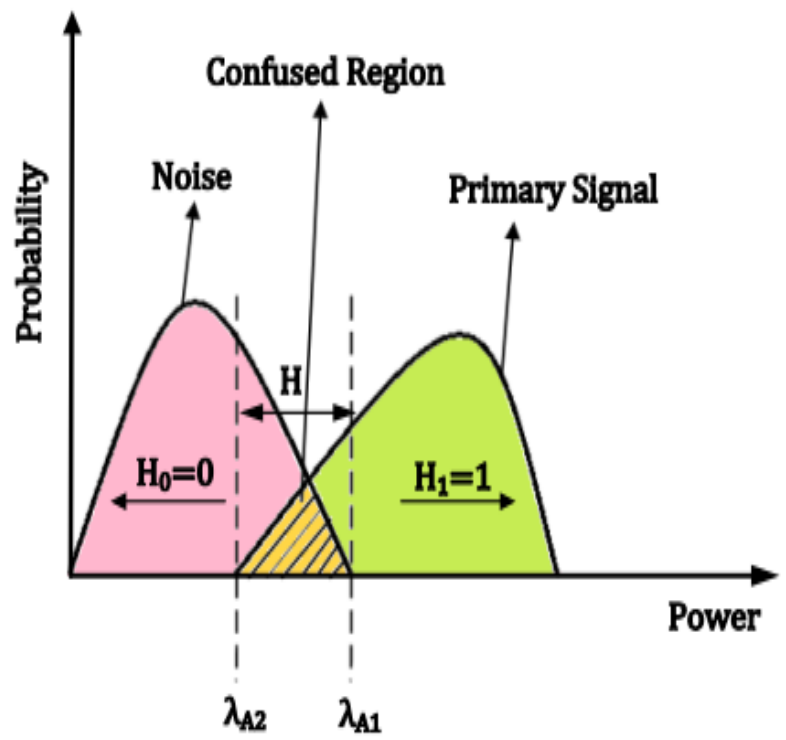

Fig.4. Energy Distribution Curve of PU Signal and noise

\section{PROPOSED FRAMEWORK}

The primary requirement for our model is Energy Detector (ED) with dynamic threshold, instead of fixed threshold with the kernel principal component analysis approach that it helps keep the probability detection higher for different SNR. Generally, PCA is used to reduce the dimensionality of data which will make spectrum sensing easier.

The received vector for d-dimensional is augmented as

$Y=\left(y(n), y(n+1), \ldots,(y(n+d-1))^{T}\right.$

$H_{0}: y=w$

$H_{1}: y=x+w$

The training set contains

$X_{1}=\left(x(n), x(n+1), \ldots \ldots,(x(n+d-1))^{T}\right.$
$X_{M}=\left(x(n)+(M-1) i, x\left(n(M-1) i+1, \ldots \ldots, x(n+(M-1) i+d-1)^{T}\right.\right.$

Where $M$ denote the number of vectors in the training set and $i$ is the sampling interval. $T$ represents transpose.

A. Proposed Kernel PCA with leading eigenvector Technique:

The key concept of this technique is that it uses eigenvector $v_{1}^{f}$ as template for spectrum sensing without need of principal components of feature space. As mentioned earlier the incorporation of kernel function in the feature space, works better as compared to the normal PCA which takes much lesser computations. Let the training set of KPCA is $\varphi\left(x_{1}\right), \varphi\left(x_{2}\right), \ldots, \varphi\left(x_{M}\right)$ has zero mean, so can be written as

$$
\frac{1}{M} \sum_{i=1}^{M} \varphi\left(x_{i}\right)=0
$$

In the similar way, the sample covariance matrix of $\varphi\left(x_{i}\right)$ is Hence the leading vector $v_{1}^{f}$ of $\mathrm{R}_{\varphi(\mathrm{x})}$ corresponding to the largest eigenvalue $\lambda_{1}^{f}$ fulfils

$R_{\varphi(x)}=\frac{1}{M} \sum_{i=1}^{M} \varphi\left(x_{i}\right) \varphi\left(x_{i}\right)^{T}$

$R_{\varphi(x)} v_{1}^{f}=\lambda_{1}^{f} v_{1}^{f}$

$=\frac{1}{M} \sum_{i=1}^{M} \varphi\left(x_{i}\right) \varphi\left(x_{i}\right)^{T} v_{1}^{f}=\lambda_{1}^{f} v_{1}^{f}$

$=\frac{1}{M} \sum_{i=1}^{M}<\varphi\left(x_{i}\right), v_{1}^{f}>\varphi\left(x_{i}\right)=\lambda_{1}^{f} v_{1}^{f}(18)$

According to eq. (18) $v_{1}^{f}$ is the linear combination of the feature space data $\varphi\left(x_{1}\right), \varphi\left(x_{2}\right), \ldots, \varphi\left(x_{M}\right)$,

$v_{1}^{f}=\sum_{i=1}^{M} \beta_{i} \varphi\left(x_{i}\right)$

Substituting (19) into (18)

$\frac{1}{M} \sum_{i=1}^{M} \varphi\left(x_{i}\right) \varphi\left(x_{i}\right)^{T} \sum_{i=1}^{M} \beta_{i} \varphi\left(x_{j}\right)=\lambda_{1}{ }^{\prime} \sum_{j=1}^{M} \beta_{j} \varphi\left(x_{j}\right)$ 
And left multiplying $\varphi\left(x_{i}\right)^{T}, t=1.2 \ldots M$ to the both sides of eq. (20), results in

$$
\begin{aligned}
& \left.\frac{1}{M} \sum_{i=1}^{M}<\varphi\left(x_{i}\right), \varphi\left(x_{i}\right)>\sum_{j=1}^{M} \beta_{j}<\varphi\left(x_{i}\right), \varphi\left(x_{j}\right)\right\rangle \\
& \left.=\lambda_{1} \sum_{j=1}^{M} \beta_{j}<\varphi\left(x_{i}\right), \varphi\left(x_{j}\right)\right\rangle
\end{aligned}
$$

By bring together the kernel matrix in to the feature space $\left.K=k\left(x_{i}, x_{j}\right)\right)_{i j}$ eq. (21), can be rewritten as

$K^{2} \beta_{1}=M \lambda_{1}^{f} K \beta_{1} \Rightarrow K \beta_{1}=M \lambda_{1}^{f} K \beta_{1}$

It can be realized that $\beta$ is the leading eigenvector of the kernel matrix $K$. The normalization of can be derived by

$$
\begin{aligned}
1 & =\left\langle v_{1}^{f}, v_{1} f\right\rangle \\
= & \left\langle\begin{array}{c}
M \\
\sum_{i=1} \beta_{i} \varphi\left(x_{i}\right), \sum_{i=1}^{M} \beta_{i} \varphi\left(x_{i}\right)
\end{array}\right\rangle \\
= & \sum_{i, j=1}^{M} \beta_{i} \beta_{j}<\varphi\left(x_{i}\right), \varphi\left(x_{j}\right)> \\
= & \beta_{1}^{T} \mathbf{K} \beta_{1} \\
= & \beta_{1}^{T} \mu_{1} \beta_{1} \\
= & \mu_{1}\left\langle\beta_{1}, \beta_{1}\right\rangle
\end{aligned}
$$

in which $\mu_{1}$ is the eigenvalue corresponding to the eigenvector $\beta_{1} 1$ of $K$. In the method [15], the primary principal component of a random point $\varphi_{(x)}$ in the feature space can be take out by

$$
\begin{aligned}
& \left\langle\varphi(x), v_{1}^{f}\right\rangle=\sum_{i=1}^{M} \beta_{i}\left\langle\varphi(x), \varphi\left(x_{i}\right)>\right. \\
& =\sum_{i=1}^{M} \beta_{i} k\left(x, x_{i}\right)
\end{aligned}
$$

Without knowing $v_{1}^{f}$ explicitly.

So in this paper, we used the efficient detection scheme explicitly with unknown $v_{1}^{f}$ simply relies on the leading eigenvector with applied covariance matrix for different feature space. The proposed algorithm can be explained with the help of flow diagram as illustrated in Fig. 5, shows that how it can detect the presence of primary user.

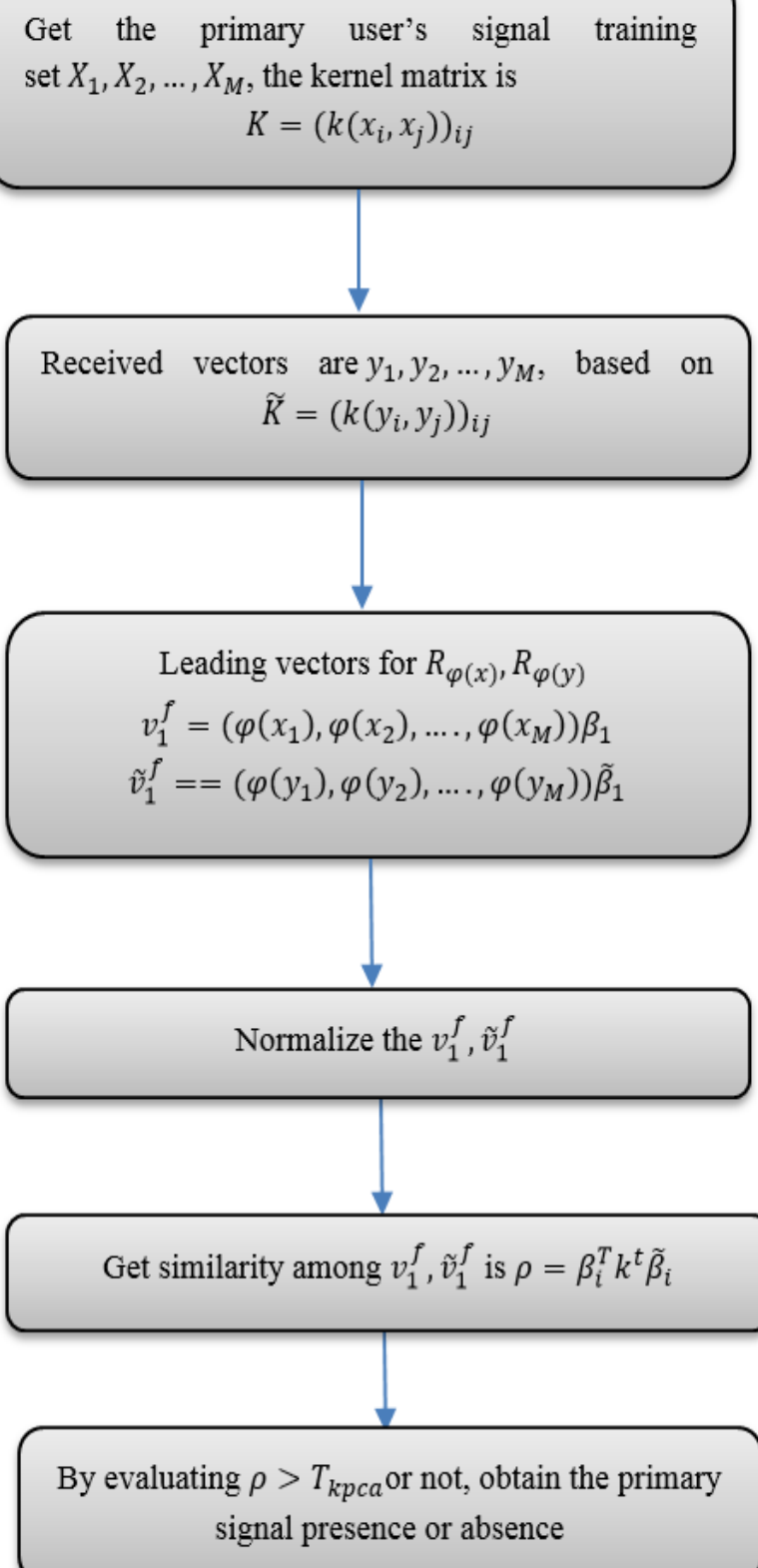

Fig. 5.Proposed kernel PCA flow diagram for spectrum sensing

\section{RESULTS AND DISCUSSION}

The simulation was done on MATLAB version R2013a through which the capability of Kernel PCA based energy detector is evaluated. Besides, the proposed system is tested by considering the parameters under various noise uncertainties for the BPSK modulation technique is used and input is the OFDM signal. Also, we express the problem of signal detection in the presence of additive noise and elucidate the spectrum detection scheme which includes Kernel PCA with the leading eigenvector-based energy detector. The first section of the OFDM signal with samples with length $L=500$ is taken as the samples of the primary user's signal $x(n)$ have taken the probability of false alarm rate supposed are $(0,1)$, and sample points of count $N=500$. 


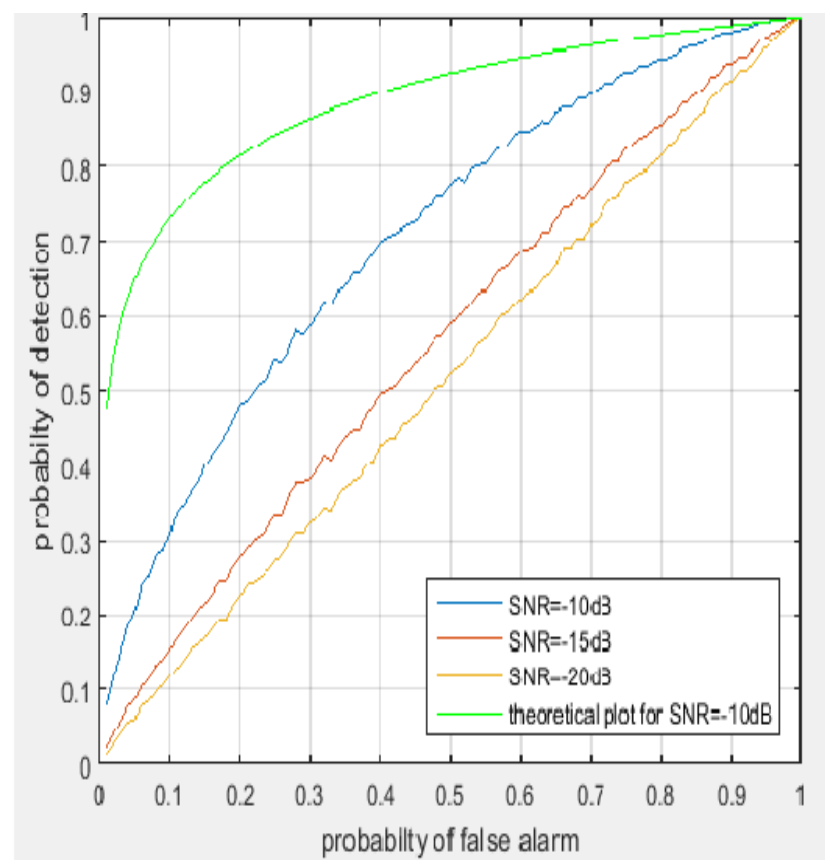

Fig.6. ROC curves for different SNR values at $N=500$

Fig. 6 clearly shows the ROC curves of PCA+ED at three different values $-20 \mathrm{~dB},-15 \mathrm{~dB},-10 \mathrm{~dB}$ to show the performance of probability of false alarm on $\mathrm{X}$-axis and probability of detection on $\mathrm{Y}$-axis. It is obvious that detection performance of the PCA based energy detector improved by increasing SNR value.

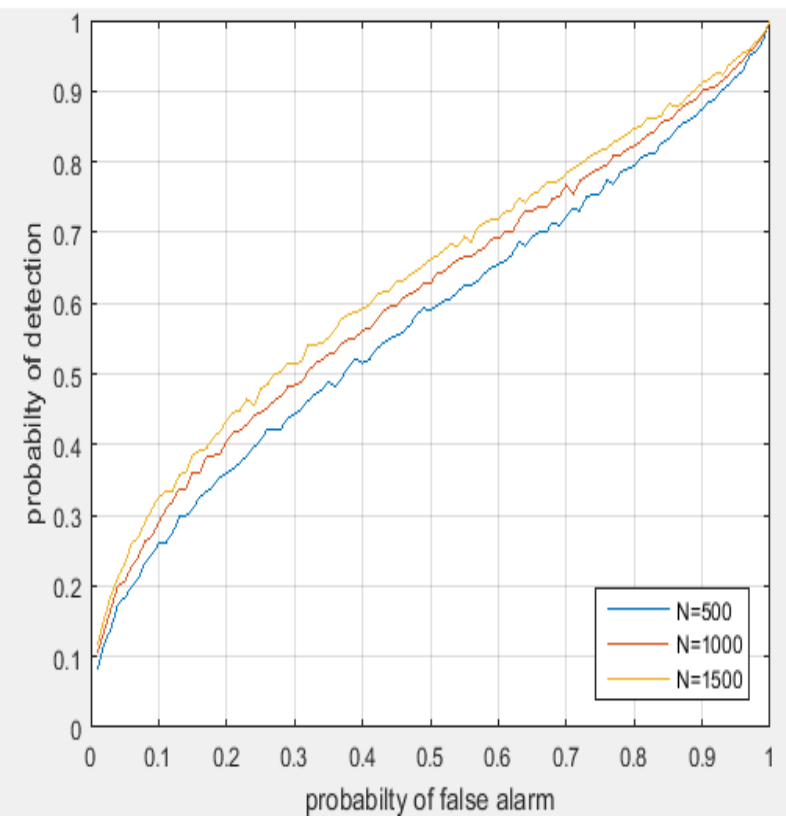

Fig.7. ROC curves for different $\mathrm{N}$ values at $\mathrm{SNR}=\mathbf{- 1 5 d B}$

Fig.7 clearly shows the ROC curves of PCA+ED at sample points taken are $\mathrm{N}=500,1000,1500$ at fixed $\mathrm{SNR}=-15 \mathrm{~dB}$ to show the performance of probability of false alarm on $\mathrm{X}$-axis and probability of detection on $\mathrm{Y}$-axis. In addition, to test, the detection performance of PCA based energy detector is possible by taking the observations for different values of false alarm values range of 0 to 1 at regular intervals to find out the probability of detection. It is obvious that the detection performance of the KPCA based energy detector enhanced for growth of $N$ (sample points) values irrespective of low SNR values.

Retrieval Number: F8703088619/2019@BEIESP

DOI: 10.35940/ijeat.F8703.129219

Journal Website: www.ijeat.org

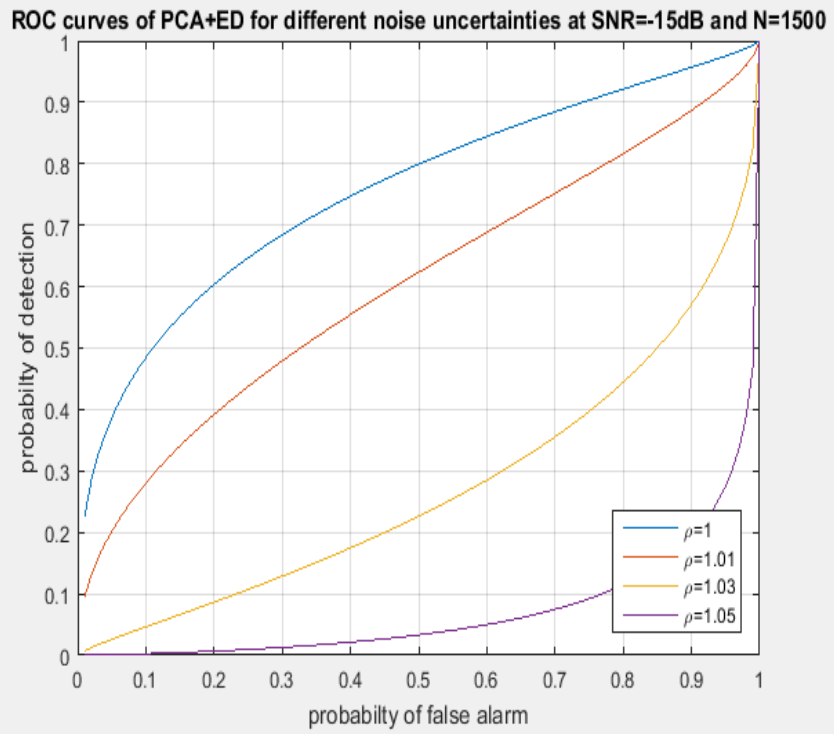

Fig. 8. ROC curves of Kernel PCA with different noise uncertainty at $N=1500$

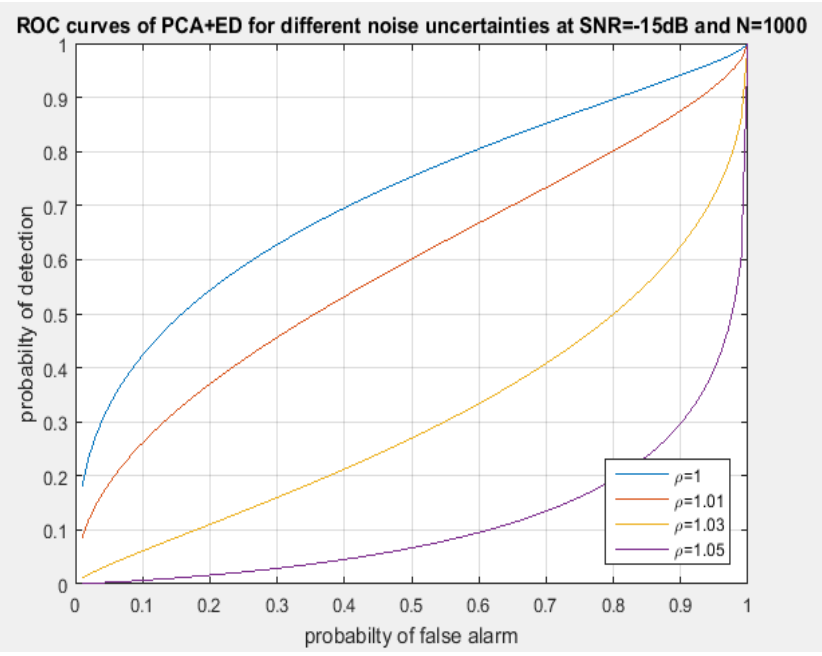

Fig.9. ROC curves of PCA+ED with different noise uncertainty at $N=1000$

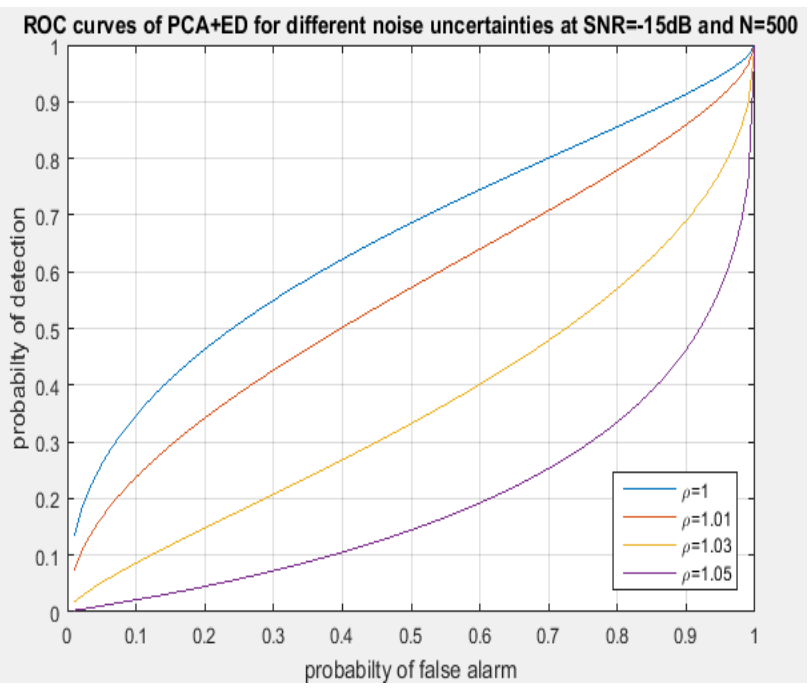

Fig. 10. ROC curves of traditional PCA+ED under various noise uncertainty at $N=500$

Published By:

Blue Eyes Intelligence Engineering \& Sciences Publication 
It is observed from Fig.8-10, there is a poor performance of the detector when traditional PCA is applied for increasing levels of noise uncertainties. Also due to the fixed threshold, there is a drastic change in the functionality of the energy detector.

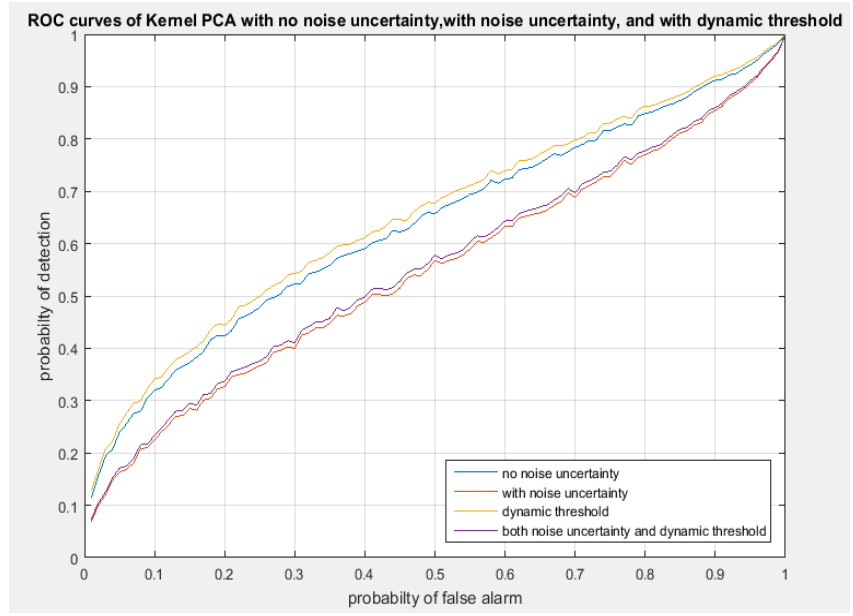

Fig.11. ROC curves of Kernel PCA+ED with different noise uncertainty at $N=\mathbf{1 5 0 0}$

Fig .11 shows the performance of our proposed Kernel PCA based energy detection scheme, in which the $P_{f a}$ on X-axis and $P_{d}$ on $\mathrm{Y}$-axis. So, therefore, introducing the dynamic threshold in place of fixed threshold for KPCA, brings about improved performance in terms of probability of detection, false alarm rates for various noise uncertainties.

\section{CONCLUSION}

The expansion of the cognitive radio network brought about remarkable changes in the industry, wireless gadgets, and other engineering applications. In this paper, to obtain the detector performance with the eigenvector ensure that an efficient model is implemented for kernel PCA. The concept associated with the inner-product between leading eigenvectors is taken as the similarity measure for the kernel PCA approach. The proposed algorithm makes the detection in an arbitrary dimensional feature space possible. In the initial stage, it is shown that to improve the performance of energy detector even at lower SNR values, the PCA scheme is applied and substantial increment in SNR values and rise in the data samples. Secondly, the performance of the proposed algorithm is evaluated under ROC curves for noise uncertainty, dynamic threshold, and both effects together. Finally, the dynamic threshold energy detection technique is combined with the kernel principal component analysis (KPCA) which given rising to improved performance as related to that of the fixed threshold mechanism.

\section{REFERENCES}

1. Wei Zhang, Member, IEEE, Ranjan K. Mallik, Senior Member, IEEE, and Khaled Ben Letaief, Fellow, IEEE "Optimization of Cooperative Spectrum Sensing with Energy Detection in Cognitive Radio Networks", IEEE transactions on wireless communications, vol. 8, no. 12, December 2009.

2. E. D. N. FCC, "03-222," Notice of Proposed Rule Making and Order, 2003.

3. Sobron, I.; Diniz, P.S.R.; Martins, W.A.; Velez, M. Energy detection technique for adaptive spectrum sensing. IEEE Trans. Commun. 2015, 63, 617-627. Yokohama, Japan, 15-18 May 2011; pp. 1-5. 3845-3857. Commun. 2015, 12, 35-44. 1099. Hyderabad, India, 2013 Cognitive Radio System,” Japan, 2016.2. Communications, China, 2015, pp. 108-121. no. 5, pp. 1299-1319, 1998. 13.

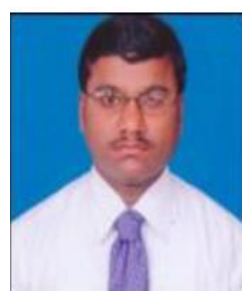
India. signal processing.

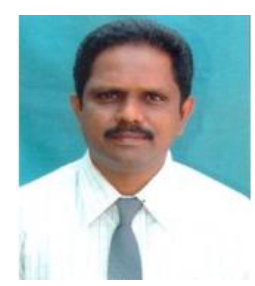
Proceedings of the IEEE Vehicular Technology Conference,

5. Del Ser, J.; Alonso, A.; Gil-Lopez, S.; Garay, M.; Kretzschmar, U.; Astarloa, A. On the Design of a Heuristically Optimized Multiband Spectrum Sensing Approach for Cognitive Radio Systems. In Proceedings of the IEEE 17th International Workshop on Computer Aided Modeling and Design of Communication Links and Networks, Barcelona, Spain, 17-19 September 2012; pp. 168-169.

6. Lee, W.Y.; Akyildiz, I.F. Optimal Spectrum Sensing Framework for Cognitive Radio Networks. IEEE Trans. Commun. 2008, 7,

7. Yang, M.; Li, Y.; Liu, X.; Tang, W. Cyclostationary feature detection-based spectrum sensing algorithm under complicated electromagnetic environment in cognitive radio networks. China

8. S.-Q. Liu, B.-J. H and X.-Y. Wang, "Hierarchical Cooperative Spectrum Sensing Based on Double Thresholds Energy Detection," IEEE Communications Letters, vol. 16, no. 7, (2012) July, pp. 1096 -

9. S. Srinu, "Entropy based Reliable Cooperative Spectrum Sensing for Cognitive Radio Networks," Doctoral dissertation, University of

10. S. Maharjan, K. Po, and J. Takada, "Energy Detector Prototype for

11. J. Mitola and G. Q. Maguire, "Cognitive radio: making software radios more personal,” IEEE Pers. Commun., vol. 6, no. 4, pp. 13-18, 1999.

12. W. Jinlong, F. Shuo, W. U. Qihui, Z. Xueqiang, and X. U. Yuhua, "Hierarchical Cognition Cycle for Cognitive Radio Networks," in

13. R. Tandra and A. Sahai, "SNR Walls for Signal Detection," Sel. Top. Signal Process. IEEE J., vol. 2, no. 1, pp. 4-17, 2008.

14. F. Bao, H. Chen, and L. Xie (2012) " Analysis of Primary User Emulation Attack with Motional Secondary Users in Cognitive Radio Networks", Proc. Of 2012 IEEE 23rd International Symposium on Personal, Indoor and Mobile Radio Communications - (PIMRC).

15. B. Scholkopf, A. Smola, and K. Muller, "Nonlinear component analysis as a kernel eigenvalue problem," Neural computation, vol. 10,

\section{AUTHORS PROFILE}

Venkatapathi Pallam, received M. Tech Degree from JNTU Hyderabad, Telangana, India in 2009.He is currently working toward the Ph.D. degree in electronics and communication engineering, KL University, Vijayawada, AP,

Also working as an Assistant Professor at Malla Reddy College of Engineering, Secunderabad, Telangana, India. He has 14 years of experience inthe field of teaching. His area of research Interest includes Wirelesscommunication \&

Dr. Habibulla Khan, received $\mathrm{Ph}$. D degree from Andhra University in the year of 2007. Presently working as Professor and dean students affaire inthe KL University. He published various nationa land international journals \& conferences. He is a member of professional bodies like IEEE, ISTE and IETE. His area of research Interest includes wireless communications \& signal processing. He published more number of papers in various national and international journals \& conferences. His area of research Interest includes wireless communications, signal processing and antennas. 


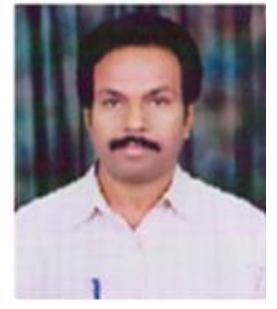

Dr. S. Srinivasa Rao, received the B.Tech degree from Madras Institute of Technology, Anna University, and the M. Tech and Ph.D fromJNTU Hyderabad, Telangana, India. Presently working as Professor and Head of the Department at Malla Reddy College of Engineering and Technology, Secunderabad. He has 26 years of experience in the field of teaching. He is a member of professional bodies like IEEE, ISTE and IETE and also reviewer for springer's international journal on wireless personal communication. $\mathrm{He}$ published more number of papers in various national and international journals \& conferences. His area of research Interest includes wireless communications \& signal processing. 